\title{
Covid-19 and gender: lower rate but same mortality of severe disease in women-an observational study
}

Federico Raimondi,2 (D), Luca Novelli , Arianna Ghirardi ${ }^{3}$, Filippo Maria Russo ${ }^{2,4}$, Dario Pellegrini ${ }^{5}$, Roberta Biza ${ }^{1,2}$, Roberta Trapasso 1,2, Lisa Giuliani 1,2, Marisa Anelli, ${ }^{1,2}$, Mariangela Amoroso ${ }^{1,2}$, Chiara Allegri ${ }^{1,2}$, Gianluca Imeri ${ }^{1}$, Claudia Sanfilippo ${ }^{1}$, Sofia Comandini ${ }^{1}$, England Hila ${ }^{2,4}$, Leonardo Manesso ${ }^{2,4}$, Lucia Gandini ${ }^{2,4}$, Pietro Mandelli ${ }^{2,4}$, Martina Monti ${ }^{2,4}$, Mauro Gori ${ }^{5}$, Michele Senni ${ }^{5}$, Ferdinando Luca Lorini ${ }^{4}$, Marco Rizzi ${ }^{6}$, Tiziano Barbui ${ }^{3}$, Laura Paris ${ }^{7}$, Alessandro Rambaldi ${ }^{2,7}$, Roberto Cosentini ${ }^{8}$, Giulio Guagliumi ${ }^{5}$, Simonetta Cesa ${ }^{9}$, Michele Colledan ${ }^{10}$, Maria Sessa ${ }^{11}$, Arianna Masciulli ${ }^{3}$, Antonello Gavazzi ${ }^{3}$, Sabrina Buoro ${ }^{12}$, Giuseppe Remuzzi ${ }^{13}$, Piero Ruggenenti ${ }^{14}$, Annapaola Callegaro ${ }^{15}$, Andrea Gianatti ${ }^{16}$, Claudio Farina ${ }^{15}$, Antonio Bellasi ${ }^{17}$, Sandro Sironi ${ }^{18,19}$, Stefano Fagiuoli ${ }^{20}$ and Fabiano Di Marco ${ }^{1,2^{*}}$ HPG23 Covid-19 Study Group

\begin{abstract}
Background: Gender-related factors might affect vulnerability to Covid-19. The aim of this study was to describe the role of gender on clinical features and 28-day mortality in Covid-19 patients.

Methods: Observational study of Covid-19 patients hospitalized in Bergamo, Italy, during the first three weeks of the outbreak. Medical records, clinical, radiological and laboratory findings upon admission and treatment have been collected. Primary outcome was 28-day mortality since hospitalization.

Results: 431 consecutive adult patients were admitted. Female patients were 119 (27.6\%) with a mean age of $67.0 \pm 14.5$ years (vs $67.8 \pm 12.5$ for males, $p=0.54$ ). Previous history of myocardial infarction, vasculopathy and former smoking habits were more common for males. At the time of admission $\mathrm{PaO}_{2} / \mathrm{FiO}_{2}$ was similar between men and women (228 [IQR, 134-273] vs $238 \mathrm{mmHg}$ [150-281], $p=0.28$ ). Continuous Positive Airway Pressure (CPAP) assistance was needed in the first $24 \mathrm{~h}$ more frequently in male patients ( $25.7 \% \mathrm{vs} 13.0 \% ; p=0.006$ ). Overall 28 -day mortality was $26.1 \%$ in women and $38.1 \%$ in men $(p=0.018)$. Gender did not result an independent predictor of death once the parameters related to disease severity at presentation were included in the multivariable analysis $(p=0.898)$. Accordingly, the Kaplan-Meier survival analysis in female and male patients requiring CPAP or non-invasive ventilation in the first $24 \mathrm{~h}$ did not find a significant difference $(p=0.687)$.
\end{abstract}

Conclusion: Hospitalized women are less likely to die from Covid-19; however, once severe disease occurs, the risk of dying is similar to men. Further studies are needed to better investigate the role of gender in clinical course and outcome of Covid-19.

Keywords: Covid-19, Gender, Disease severity

*Correspondence: fabiano.dimarco@unimi.it

${ }^{1}$ Pulmonary Medicine Unit, Medicine Department, ASST Papa Giovanni XXIII, Piazza OMS, 1, 24127 Bergamo, Italy

Full list of author information is available at the end of the article

\section{Background}

Severe Acute Respiratory Syndrome Coronavirus (SARSCoV-2) infection disease (Covid-19) was first described in 
December 2019 in Whuan, China. As of 12 March 2020, due to the growing number of countries involved, the World Health Organization (WHO) declared Covid-19 a pandemic [1]. Northern Italy has been one of the first and most severely affected area of Europe, with an increase in mortality up to $+568 \%$ in Bergamo and its Province (over 1 million inhabitants), in March 2020 compared to the same month in 2015-2019 [2].

A growing body of literature on SARS-CoV-2 infection addressing immediate biomedical needs (e.g. clinical characteristics, mortality, and predictors of outcome) is becoming available, however only few articles specifically refer to the gender dimension of Covid-19 [3-7]. In the 2003 Severe Acute Respiratory Syndrome (SARS, caused by SARS-CoV-1 infection) epidemic, sex differences indicated a lower risk of death in women [8]. Analogous observations can be made for SARS-CoV-2 infection for which, compared to women, men have a higher mortality risk [7, 9-12]. These results suggest an underlying sexdependent susceptibility. An enzymatic system involved in this different sex predisposition could be represented by angiotensin converting enzyme 2 (ACE2), which is the functional receptor for SARS-CoV-1 and 2 [13]. In animal experimental models ACE2 expression is found to be influenced by sex hormones; in female mice, ovariectomy or administration of estrogen-receptor antagonists increased the mortality of SARS-CoV-1 infection [14]. Furthermore, women have stronger innate and adaptive immunity and overall greater resistance to viral infections than men [15]. As emphasized for previous outbreaks of global concern (i.e. MERS, H1N1, H5N1, SARS, Zika and Ebola virus), the study of gender dimension, which is both physical and social constructed, is important to understand the pathogenic mechanisms and to eventually design better therapeutic strategies [16].

The aim of this study was to describe the role of gender in terms of clinical features and 28-day outcomes of hospitalized Covid-19 patients.

\section{Methods}

This retrospective, observational study was approved by the local Ethics Committee (Comitato Etico di Bergamo, Italy. $\left.\mathrm{N}^{\circ} 37 / 2020\right)$. In the light of the urgent need to treat critical patients, and to avoid paper contamination, verbal consent was obtained when feasible, according to local protocol.

\section{Source of data}

We collected data from electronic medical records of all adult patients with laboratory-confirmed SARS-CoV-2 infection, consecutively hospitalized for clinical reasons (i.e. respiratory failure in almost all cases) at Papa Giovanni XXIII Hospital (a tertiary hospital of 1080 beds), and its affiliate hospital, San Giovanni Bianco (a community hospital of 130 beds), between February 23rd and March 14th, 2020. Follow-up stopped on April 11th, 2020 , to allow the observation for a minimum of 28 days in all patients since hospitalization. We did not include patients under eighteen year-old or patients already hospitalized for other conditions. Covid-19 has been diagnosed on the basis of the updated WHO interim guidance [17]. Medical history, demographic data, underlying comorbidities, viral exposure, clinical symptoms and/or signs, radiological and laboratory findings upon admission were derived from medical records, whereas information about family unit, healthcare job, prehospital medical contact, use of antibiotics and flu vaccine status were self-reported by the patient or relatives. Immunosuppression was defined as iatrogenic when due to chemotherapy, or treatment for solid organ transplantation or autoimmune diseases, otherwise it was HIVrelated (Human Immunodeficiency Virus). Radiologic assessments and all laboratory tests were performed according to local clinical practice and based on clinical needs. At presentation, patients underwent routine blood tests, arterial blood gas analysis (ABG), and chest X-ray.

\section{Laboratory confirmation of SARS-CoV-2 infection}

SARS-CoV-2 genome from nasal swabs and respiratory samples was detected by two different molecular methods (GeneFinder COVID-19-Elitech Group, Allplex ${ }^{\text {TM }}$ 2019- $\mathrm{nCoV}$ Assay-Seegene Inc) in line with the manufacturer's instructions. After the purification of viral RNA from clinical samples, the detection of RdRp, E and $\mathrm{N}$ viral genes was obtained by real time Polymerase Chain Reaction (RT-PCR) according to WHO protocol [18].

\section{Outcomes}

The aim of this study was to describe gender differences in terms of clinical features and 28-day outcomes since hospitalization. The primary endpoint was 28-day all-cause mortality, occurring either during in-hospital stay or after discharge. The secondary endpoint was the development of severe disease, a composite outcome defined as the occurrence of at least one of the following: intensive or respiratory sub-intensive care unit admission; need of endotracheal intubation (ETI) and invasive ventilation, non-invasive ventilation (NIV), or continuous positive airway pressure (CPAP); death during hospitalization or after discharge.

\section{Statistical analysis}

Descriptive statistics were used to summarize the baseline characteristics of Covid-19 patients. Continuous 
variables were expressed as mean \pm standard deviation (SD) or as median and interquartile range [IQR], depending on their parametric or non-parametric distribution. Categorical variables were expressed as absolute counts and percentages. The chi-square test (or Fisher's exact test when appropriate) was used to test between group differences for the categorical variables, whereas the t-test or the Wilcoxon-Mann-Whitney test (for normally and not normally distributed variables, respectively) were used to compare continuous variables. Survival curve (overall and stratified by the need of CPAP/NIV at entry), according to gender was reported, with comparison by the log-rank test. Univariate logistic regression model was run to investigate predictors of 28-day mortality. A backward stepwise procedure was used to determine the best predictors of mortality to be included in the multivariable model. Results are presented as odds ratio (OR) with $95 \%$ confidence intervals (CI). Candidate predictors included in the stepwise procedure were variables that were available in at least $65 \%$ of patients and significantly different between patients who died and those who did not at a $p$ value level of 0.05 . The final multivariable model included predictors selected from the stepwise procedure along with some few other variables selected on biological plausibility and clinical judgment. To overcome the constraint of biased/overestimated results that may arise as a result of missing data, multiple imputation by chained equation (MICE), with $20 \mathrm{impu-}$ tation sets, was used to impute the missing covariates involved in the final multivariable model. For all tested hypotheses, a two-tailed $p$ values $<0.05$ was considered significant. Analyses were performed using STATA software, release 16 (StataCorp LP, College Station, TX, USA).

\section{Results}

In the first three weeks of the pandemic 431 adult Covid-19 patients were admitted by the ER to our hospital. Their demographic and clinical characteristics are reported in Table 1. 119 were female (27.6\%), and the majority were Caucasian (98.6\%). The mean $( \pm S D)$ age was $67.6 \pm 13.0$ years, notably no significant differences were observed between female and male $(67.0 \pm 14.5 \mathrm{vs}$ $67.8 \pm 12.5$ years, respectively, $p=0.54$ ). Regardless of gender, most patients had documented relevant comorbidities, especially systemic hypertension (55.8\%), and diabetes (19.8\%). However, being a former smoker, a previous history of myocardial infarction or vasculopathy were significantly more common in males $(33.3 \% \mathrm{vs}$ $20.5 \%, 16.1 \%$ vs $3.4 \%, 15.4 \%$ vs $6.8 \%$; $p=0.025, p<0.001$, $p=0.020$; respectively). The comorbidity burden, as estimated by the Charlson Comorbidity Index (CCI), attained a median value of 4.0 [IQR 2.0-5.0] and was comparable between categories as well as medication history, immunosuppression and flu vaccine status.

\section{Pre-hospital, clinical and laboratory features at presentation}

Pre-hospital epidemiology, in terms of contact with healthcare facilities or infection occurrence within family unit, was homogeneous among groups (Table 2). Half of the patients had taken antibiotics before hospitalization and fever was the most frequent symptom at home $(90.1 \%)$, followed by dyspnea $(59.3 \%)$ and cough (50.2\%). Interestingly, gastrointestinal symptoms (i.e. anorexia, nausea, vomiting, diarrhea) were significantly more prevalent in females $(24.6 \%$ vs $15.7 \% ; p=0.033)$. Relevant intervals between symptoms onset and clinically important episodes are reported in Additional file 1: Table S1. The median interval between symptoms onset and ER admission resulted of 7.0 days [5.0-10.0], without significant difference according to gender $(8.0$ [5.0-10.5] vs 7.0 [5.0-10.0]) in females and males, respectively, $p=0.97$ ). Of note, interval between hospitalization and CPAP/NIV treatment was significantly shorter in males ( 1 day [1-3] vs 2 days [1-4]; $p=0.017$ ).

Characteristics at presentation to ER are shown in Table 3. Most of the patients were alert (93.8\%), and febrile $(65.2 \%)$, with normal blood pressure and normal heart rate. ABG showed for both sexes a tendency to respiratory alkalosis, with a median $\mathrm{pH}$ of 7.47 [7.447.50], median $\mathrm{PaCO}_{2}$ of $33 \mathrm{mmHg}$ [IQR 30-35], and median $\mathrm{HCO}_{3}{ }^{-}$of $24.1 \mathrm{mmol} / \mathrm{l}$ [22.0-26.0]. Females showed a small but significant higher heart rate and $\mathrm{HCO}_{3}{ }^{-}$(Table 3). Also $\mathrm{PaO}_{2} / \mathrm{FiO}_{2}$ at admission, on average severely reduced, was not statistically different between male and female $(229 \mathrm{mmHg}$ [134-273] vs $238 \mathrm{mmHg}$ [150-281], $p=0.28)$.

Laboratory and radiographic findings at admission are reported in Table 4. Liver transaminases, both AST and ALT, were higher in males than in females (53 U/L vs $40 \mathrm{U} / \mathrm{L}$ and $40 \mathrm{U} / \mathrm{L}$ vs $30 \mathrm{U} / \mathrm{L}$, respectively; $p<0.001$ ). Urea and creatinine values were normal, although significantly lower in female $(0.74 \mathrm{mg} / \mathrm{dL} v s 0.98 \mathrm{mg} / \mathrm{dL}$ and $38 \mathrm{mg} / \mathrm{dL}$ vs $48 \mathrm{mg} / \mathrm{dL}$, respectively; $p<0.001$ ). CRP was generally elevated (113 [56-162] $\mathrm{mg} / \mathrm{L})$, with cases of very high levels (i.e. $>127 \mathrm{mg} / \mathrm{L}$ ) being more common in males (35.4 vs $48.7 \%, p$ 0.015). Procalcitonin (PCT) levels were higher in males $(0.56 \mathrm{ng} /$ $\mathrm{mL}$ [IQR $0.21-2.35$ ] vs $0.12 \mathrm{ng} / \mathrm{mL}$ [IQR $0.05-1.00$ ]; $p=0.019)$. The complete blood count, coagulation parameters, liver and renal function, although differed from gender categories, in the majority of cases were in the range of normality. The overall prevalence of chest 
Table 1 Demographic and clinical characteristics in all patients and by gender

\begin{tabular}{|c|c|c|c|c|c|}
\hline & \multirow[t]{2}{*}{ N } & \multirow[t]{2}{*}{ All patients $(\mathrm{N}=431)$} & \multicolumn{3}{|l|}{ Gender } \\
\hline & & & Female $(\mathrm{N}=119)$ & Male $(N=312)$ & $p$ \\
\hline Age-y mean (SD) & 431 & $67.6(13.0)$ & $67.0(14.5)$ & $67.8(12.5)$ & 0.54 \\
\hline$\leq 59-$ no. $(\%)$ & 431 & $109(25.3)$ & $32(26.9)$ & $77(24.7)$ & 0.29 \\
\hline 60-69-no. (\%) & & $103(23.9)$ & $24(20.2)$ & $79(25.3)$ & \\
\hline 70-77-no. (\%) & & $114(26.5)$ & $38(31.9)$ & $76(24.4)$ & \\
\hline$\geq 78-$ no. (\%) & & $105(24.4)$ & $25(21.0)$ & $80(25.6)$ & \\
\hline Caucasian ethnicity—no. (\%) & 431 & $425(98.6)$ & $118(99.2)$ & $307(98.4)$ & 0.55 \\
\hline $\mathrm{BMI}^{\mathrm{a}}$-median $[\mathrm{IQR}]$ & 359 & $26.8[24.5-30.2]$ & $26.3[22.8-31.2]$ & $27.0[24.8-30.1]$ & 0.14 \\
\hline$\geq 30$-no. (\%) & 359 & $97(27.0)$ & $30(30.9)$ & $67(25.6)$ & 0.31 \\
\hline \multicolumn{6}{|l|}{ Smoking history—no. (\%) } \\
\hline Current smoker & 319 & $18(5.6)$ & $4(4.5)$ & $14(6.1)$ & 0.60 \\
\hline Former smoker & 319 & $95(29.8)$ & $18(20.5)$ & $77(33.3)$ & 0.025 \\
\hline Never smoker & 319 & $206(64.6)$ & $66(75.0)$ & $140(60.6)$ & 0.016 \\
\hline \multicolumn{6}{|l|}{ Comorbidities—no. (\%) } \\
\hline Hypertension & 425 & $237(55.8)$ & $61(51.7)$ & $176(57.3)$ & 0.29 \\
\hline Diabetes & 425 & $84(19.8)$ & $18(15.3)$ & $66(21.5)$ & 0.15 \\
\hline Chronic Kidney Failure & 423 & $31(7.3)$ & $9(7.8)$ & $22(7.2)$ & 0.83 \\
\hline COPD & 423 & $41(9.7)$ & $13(11.1)$ & $28(9.2)$ & 0.54 \\
\hline Active solid neoplasm & 423 & $16(3.8)$ & $6(5.1)$ & $10(3.3)$ & 0.37 \\
\hline Active hematologic malignancy & 423 & $11(2.6)$ & $2(1.7)$ & $9(2.9)$ & 0.48 \\
\hline Cerebrovascular disease & 422 & $25(5.9)$ & $6(5.1)$ & $19(6.2)$ & 0.67 \\
\hline Previous Myocardial Infarction & 422 & $53(12.6)$ & $4(3.4)$ & $49(16.1)$ & $<0.001$ \\
\hline Chronic heart failure & 423 & $16(3.8)$ & $5(4.3)$ & $11(3.6)$ & 0.74 \\
\hline Vasculopathy & 423 & $55(13.0)$ & $8(6.8)$ & $47(15.4)$ & 0.020 \\
\hline Rheumatic pathology & 423 & $28(6.6)$ & $9(7.7)$ & $19(6.2)$ & 0.58 \\
\hline CCl score-median [IQR] & 426 & $4.0[2.0-5.0]$ & $4.0[2.0-5.0]$ & $4.0[2.0-5.0]$ & 0.27 \\
\hline $\mathrm{CCl}=0-$ no. (\%) & 426 & $36(8.5)$ & $13(10.9)$ & $23(7.5)$ & 0.41 \\
\hline $\mathrm{CCl}=1-2-$ no. (\%) & & $102(23.9)$ & $25(21.0)$ & $77(25.1)$ & \\
\hline $\mathrm{CCl}=3+-$ no. $(\%)$ & & $288(67.6)$ & $81(68.1)$ & $207(67.4)$ & \\
\hline \multicolumn{6}{|l|}{ Medication history—no. (\%) } \\
\hline Antihypertensives & 412 & $224(54.4)$ & $60(52.2)$ & $164(55.2)$ & 0.58 \\
\hline ACE-inhibitors & 416 & $73(17.5)$ & $21(18.4)$ & $52(17.2)$ & 0.77 \\
\hline ARBs & 416 & $72(17.3)$ & $13(11.4)$ & $59(19.5)$ & 0.050 \\
\hline Steroids & 415 & $20(4.8)$ & $8(6.9)$ & $12(4.0)$ & 0.22 \\
\hline Oral antidiabetics & 415 & $59(14.2)$ & $16(13.8)$ & $43(14.4)$ & 0.88 \\
\hline Insulin & 415 & $27(6.5)$ & $5(4.3)$ & $22(7.4)$ & 0.26 \\
\hline OAT/DOACs & 413 & $51(12.3)$ & $18(15.7)$ & $33(11.1)$ & 0.20 \\
\hline Antiplatelets & 415 & $112(27.0)$ & $28(24.1)$ & $84(28.1)$ & 0.42 \\
\hline Long-term oxygen therapy & 423 & $10(2.4)$ & $4(3.4)$ & $6(2.0)$ & 0.38 \\
\hline Immunosuppression—no. (\%) & 423 & $27(6.4)$ & $9(7.7)$ & $18(5.9)$ & 0.50 \\
\hline latrogenic & 423 & $27(6.4)$ & $9(7.7)$ & $18(5.9)$ & 0.51 \\
\hline HIV & 423 & $0(0.0)$ & $0(0.0)$ & $0(0.0)$ & - \\
\hline Flu vaccine-no. (\%) & 290 & $142(49.0)$ & $35(42.7)$ & $107(51.4)$ & 0.18 \\
\hline
\end{tabular}

Data expressed as column percentages. Percentages may not total 100 because of rounding. BMI= Body Mass Index

a Body Weight and Height as referred by patient; $\mathrm{COPD}=$ Chronic Obstructive Pulmonary Disease; $\mathrm{CCl}=\mathrm{Charlson}$ Comorbidity Index score; Antihypertensives: (ACEinhibitors, ARBs [Angiotensin Receptor Blockers], Calcium channels blockers, Diuretics, Beta-blockers, Alpha-blockers, Alpha-2 agonists); OAT = Oral Anticoagulant Therapy; DOACs = Direct Oral Anticoagulants; latrogenic immunosuppression (due to chemotherapy, solid organ transplantation, autoimmune diseases), HIV (Human Immunodeficiency Virus); SD = Standard Deviation; IQR = Interquartile Range; $p$ values obtained by Chi-square test (or Fisher's exact test when appropriate) for categorical variables and t-test (or Wilcoxon-Mann-Whitney test when appropriated) for continuous variables 
Table 2 Pre-hospital epidemiology and clinical features in all patients and by gender

\begin{tabular}{|c|c|c|c|c|c|}
\hline & \multirow[t]{2}{*}{$\mathrm{N}$} & \multirow[t]{2}{*}{ All patients $(\mathrm{N}=431)$} & \multicolumn{3}{|l|}{ Gender } \\
\hline & & & Female $(\mathrm{N}=119)$ & Male $(N=312)$ & $p$ \\
\hline Pre-hospital antibiotic ${ }^{\mathrm{a}}$ —no. (\%) & 384 & $192(50.0)$ & $49(45.4)$ & $143(51.8)$ & 0.26 \\
\hline Contact with healthcare facilities in the last 14 days ${ }^{b}$ —no. (\%) & 380 & $125(32.9)$ & $40(36.4)$ & $85(31.5)$ & 0.36 \\
\hline Contact with minors in the last 14 days-no. (\%) & 271 & $99(36.5)$ & $33(41.8)$ & $66(34.4)$ & 0.25 \\
\hline Family members ${ }^{c}$, median $[\mathrm{IQR}]$ & 295 & $2.0[2.0-3.0]$ & $2.0[2.0-3.0]$ & $2.0[2.0-3.0]$ & 0.85 \\
\hline Family member with confirmed Covid-19—no. (\%) & 296 & $45(15.2)$ & $15(17.6)$ & $30(14.2)$ & 0.46 \\
\hline Contact with Covid-19 confirmed case - no. (\%) & 317 & $81(25.6)$ & $21(22.6)$ & $60(26.8)$ & 0.43 \\
\hline Healthcare professional—no. (\%) & 380 & $27(7.1)$ & $8(7.5)$ & $19(7.0)$ & 0.86 \\
\hline \multicolumn{6}{|l|}{ Symptoms ${ }^{\mathrm{d}}$-no. (\%) } \\
\hline Fever & 425 & $383(90.1)$ & $107(90.7)$ & $276(89.9)$ & 0.81 \\
\hline Cough & 424 & $213(50.2)$ & $62(52.5)$ & $151(49.3)$ & 0.56 \\
\hline Dyspnoea & 425 & $252(59.3)$ & $68(57.6)$ & $184(59.9)$ & 0.66 \\
\hline Sore throat & 424 & $13(3.1)$ & $6(5.1)$ & $7(2.3)$ & 0.20 \\
\hline Dizziness & 424 & $18(4.2)$ & $9(7.6)$ & $9(2.9)$ & 0.032 \\
\hline Abdominal pain & 424 & $10(2.4)$ & $5(4.2)$ & $5(1.6)$ & 0.11 \\
\hline Chest pain & 424 & $15(3.5)$ & $3(2.5)$ & $12(3.9)$ & 0.49 \\
\hline Systemic (asthenia, myalgia) & 424 & $137(32.3)$ & $45(38.1)$ & $92(30.1)$ & 0.11 \\
\hline Gastrointestinal $^{e}$ & 424 & $77(18.2)$ & $29(24.6)$ & $48(15.7)$ & 0.033 \\
\hline
\end{tabular}

Data expressed as column percentages. Percentages may not total 100 because of rounding

a Pre-hospital antibiotic therapy prescribed for onset of symptoms related to Covid-19

${ }^{b}$ Access to healthcare facilities (i.e. outpatient clinic consultations, dialysis, previous hospitalization, assistance or visits to hospitalized or in retirement home people)

c Family member number intended as cohabitants

d Symptoms referred by patients to the Emergency Room (ER) as described in admission dossier

e Gastrointestinal symptoms include anorexia, nausea, vomiting and diarrhea. IQR=Interquartile Range; $p$ values obtained by Chi-square test (or Fisher's exact test when appropriate) for categorical variables

X-ray abnormalities at presentation were comparable in men and women, as well as the unilateral or bilateral onset of viral pneumonia (Table 4).

\section{Respiratory support in the first $24 \mathrm{~h}$ and in-hospital treatment}

The type of support used to treat respiratory failure in the first $24 \mathrm{~h}$ and in-hospital treatment are shown in Table 3. In order to correct hypoxemia, $34.8 \%(n=40)$ of women required low flow oxygen nasal cannula, whereas in men this treatment was sufficient in a lower percentage (i.e. 23.7\% [ $\mathrm{n}=71], p=0.022$ ). Indeed, males necessitated CPAP more frequently than females at presentation $(25.7 \%$ vs $13.0 \%$; $p=0.006)$. Considering in-hospital treatments, no gender differences were observed, with the exception of IL-6 pathway inhibitors that were used only in 17 males.

\section{Clinical outcomes and gender as predictor of mortality}

Overall 28-day mortality occurred in $34.8 \%$ of the patients (150/431), of whom 26.1\% (31/119) were women and $38.1 \%(119 / 312)$ were men $(p=0.018$,
Additional file 1: Table S2). Kaplan-Meier survival curve at 28-day (Fig. 1) found a lower mortality for females $(p=0.021)$. The secondary outcome (i.e. development of severe disease) were reported in the $59.9 \%$ of the patients (258/431), and it occurred more frequently in male patients $(63.1 \%(197 / 431)$ vs $51.3 \%(61 / 11)$; $p=0.024$, Additional file 1: Table S2). When outcomes were stratified by age categories, no gender differences were noted $(p=0.091$ and $p=0.052$ for primary and secondary outcome, respectively, Additional file 1 : Table S2).

Multivariable analysis aimed at evaluating independent predictors of mortality is shown in Table 5. When baseline demographic, clinical characteristics and prehospital epidemiologic and clinical features emerged by univariate analysis were included in the multivariable analysis, male sex, together with older age, immunosuppression and dyspnea resulted independent predictors of death. However, once the parameters related to the severity of disease at presentation (i.e. need of CPAP or NIV in the first $24 \mathrm{~h}$, and $\mathrm{PaO}_{2} / \mathrm{FiO}_{2}<200 \mathrm{mmHg}$ at admission) were included in the model, gender did not result an independent predictor of death $(p=0.898$, Table 5$)$. 
Table 3 Clinical characteristics, blood gas analysis at presentation and in-hospital treatments in all patients and by gender

\begin{tabular}{|c|c|c|c|c|c|}
\hline & \multirow[t]{2}{*}{$\mathrm{N}$} & \multirow[t]{2}{*}{ All patients $(N=431)$} & \multicolumn{3}{|l|}{ Gender } \\
\hline & & & Female $(\mathrm{N}=119)$ & Male $(\mathrm{N}=312)$ & $p$ \\
\hline \multicolumn{6}{|l|}{ At entry in emergency room } \\
\hline AVPU—no. (\%) & 421 & & & & \\
\hline A (alert) & & $395(93.8)$ & $109(93.2)$ & $286(94.1)$ & 0.39 \\
\hline V (verbal) & & $4(1.0)$ & $2(1.7)$ & $2(0.7)$ & \\
\hline$P$ (pain) & & $4(1.0)$ & $2(1.7)$ & $2(0.7)$ & \\
\hline U (unresponsive) & & $18(4.3)$ & $4(3.4)$ & $14(4.6)$ & \\
\hline HR, bpm—median [IQR] & 400 & 84 [75-94] & 87 [78-95] & 83 [73-93] & 0.024 \\
\hline $\mathrm{SBP}, \mathrm{mmHg}$-median [IQR] & 395 & $126[112-140]$ & $126[113-140]$ & $126[110-140]$ & 0.82 \\
\hline RR, acts/min—median [IQR] & 221 & $20[16-26]$ & $20[16-26]$ & $20[16-26]$ & 0.35 \\
\hline Fever-no. (\%) & 405 & $264(65.2)$ & $69(61.1)$ & $195(66.8)$ & 0.28 \\
\hline $\mathrm{pH}$-median [IQR] & 277 & $7.47[7.44-7.50]$ & $7.49[7.44-7.51]$ & $7.47[7.44-7.50]$ & 0.16 \\
\hline $\mathrm{PaO}_{2} / \mathrm{FiO}_{2}$-median [IQR] & 295 & $229[142-278]$ & $238[150-281]$ & 229 [134-273] & 0.28 \\
\hline$<200-$ no. $(\%)$ & 295 & $113(38.3)$ & $23(32.9)$ & $90(40.0)$ & 0.28 \\
\hline $\mathrm{PaCO}_{2}, \mathrm{mmHg}$-median [IQR] & 292 & $33.0[29.7-35.0]$ & $32.0[30.0-36.2]$ & $33.0[29.0-35.0]$ & 0.62 \\
\hline $\mathrm{HCO}_{3}^{-},{ }^{-}, \mathrm{mmol} / \mathrm{L}-$ median $[\mathrm{IQR}]$ & 151 & $24.1[22.0-26.0]$ & $25.4[23.4-27.4]$ & $24.0[22.0-25.0]$ & $<0.001$ \\
\hline Lac, mmol/L—median [IQR] & 188 & $1.38[1.01-1.74]$ & $1.26[1.00-1.62]$ & $1.40[1.03-1.88]$ & 0.27 \\
\hline \multicolumn{6}{|l|}{ In the first $24 \mathrm{~h}$} \\
\hline \multicolumn{6}{|c|}{ Oxygen and ventilatory support ${ }^{\mathrm{a}}$-no. (\%) } \\
\hline Low flow oxygen nasal cannula & 415 & $111(26.7)$ & $40(34.8)$ & $71(23.7)$ & 0.022 \\
\hline Venturi mask & 415 & $50(12.0)$ & $11(9.6)$ & $39(13.0)$ & 0.34 \\
\hline Non-rebreather mask & 415 & $95(22.9)$ & $27(23.5)$ & $68(22.7)$ & 0.86 \\
\hline CPAP & 415 & $92(22.2)$ & $15(13.0)$ & $77(25.7)$ & 0.006 \\
\hline NIV & 415 & $14(3.4)$ & $2(1.7)$ & $12(4.0)$ & 0.25 \\
\hline ETI & 415 & $12(2.9)$ & $4(3.5)$ & $8(2.7)$ & 0.74 \\
\hline $\mathrm{FiO}_{2}$-median [IQR] & 373 & $60.0[35.0-70.0]$ & $50.0[30.0-70.0]$ & $60.0[35.0-70.0]$ & 0.025 \\
\hline PEEP, $\mathrm{cmH}_{2} \mathrm{O}$-median [IQR] & 109 & $15.0[12.0-16.0]$ & $15.0[13.0-15.5]$ & $15.0[12.0-16.0]$ & 0.67 \\
\hline IPAP, $\mathrm{cmH}_{2} \mathrm{O}$-median [IQR] & 15 & $20.0[16.0-24.0]$ & $21.0[20.0-22.0]$ & $20.0[16.0-24.0]$ & 0.86 \\
\hline Antiviral ${ }^{b}$ —no. (\%) & 399 & $326(81.7)$ & $83(76.9)$ & $243(83.5)$ & 0.13 \\
\hline Hydroxychloroquine-no. (\%) & 392 & $305(77.8)$ & $81(76.4)$ & $224(78.3)$ & 0.69 \\
\hline Steroid ${ }^{c}-$ no. (\%) & 395 & $39(9.9)$ & $6(5.6)$ & $33(11.5)$ & 0.083 \\
\hline Antibiotics ${ }^{\mathrm{d}}$-no. (\%) & 406 & $372(91.6)$ & $103(92.0)$ & $269(91.5)$ & 0.88 \\
\hline IL-6 inhibitors ${ }^{\mathrm{e}}$-no. (\%) & 387 & $17(4.4)$ & $0(0.0)$ & $17(6.0)$ & 0.005 \\
\hline
\end{tabular}

Data expressed as column percentages. Percentages may not total 100 because of rounding. AVPU = level of consciousness; $H R=H e a r t$ Rate; RR $=$ Respiratory Rate; $\mathrm{SBP}=$ Systolic Blood Pressure; $\mathrm{ER}=$ Emergency Room; $\mathrm{FiO}_{2}=$ Fraction of inspired oxygen; $\mathrm{PaO}_{2}=$ Partial pressure of oxygen in arterial blood; $\mathrm{SaO}_{2}=\mathrm{Oxygen}$ arterial Saturation; $\mathrm{PaCO}_{2}=$ Partial pressure of Carbon Dioxide in arterial blood; $\mathrm{HCO}_{3}{ }^{-}=$Bicarbonate; Lac = Lactate concentration in arterial blood; PEEP = Positive End Expiratory Pressure; IPAP = Inspiratory Positive Airway Pressure (Pressure support + PEEP)

a Oxygen and Ventilatory support in the first $24 \mathrm{~h}$ by the ER presentation intended as the highest between Low flow oxygen nasal cannula, Venturi mask, Nonrebreather mask (reservoir), Continuous Positive Airway Pressure (CPAP) with helmet, Non-invasive ventilation (NIV) and Endotracheal Intubation (ETI)

b Antiviral therapy intended as at least one of the following: Oseltamivir, Lopinavir/Ritonavir, Remdesivir and Darunavir/Cobicistat

c Steroid therapy intended as Methylprednisolone, Hydrocortisone and Dexamethasone

d Antibiotic Therapy was prescribed at Physician's discretion and at least one of the following: Ceftriaxone, Cefixime, Azithromycin or Levofloxacin

e IL-6 inhibitors, intended as Tocilizumab or Siltuximab, were prescribed in according to shared local clinical protocol. IQR $=$ Interquartile Range; $p$ values obtained by Chi-square test (or Fisher's exact test when appropriate) for categorical variables and Wilcoxon-Mann-Whitney test for continuous variables

Accordingly, Kaplan-Meier survival analysis at 28-day in patients who needed CPAP or NIV in the first $24 \mathrm{~h}$ did not find a significant difference between men and women (Fig. 2, $p=0.687$ ).

\section{Discussion}

The main results from this study, aimed at evaluating the role of gender in Covid-19 hospitalized patients, can be summarized as follows. First, women are less prevalent than men in our setting, representing about 
Table 4 Laboratory and radiographic findings at hospital admission in all patients and by gender

\begin{tabular}{|c|c|c|c|c|c|}
\hline & \multirow[t]{2}{*}{$N$} & \multirow[t]{2}{*}{ All patients $(\mathrm{N}=431)$} & \multicolumn{3}{|l|}{ Gender } \\
\hline & & & Female $(\mathrm{N}=119)$ & Male $(N=312)$ & $p$ \\
\hline Hemoglobin, g/L & 417 & 134 [123-148] & 127 [117-136] & 139 [126-150] & $<0.001$ \\
\hline White blood cells, $10^{9} / \mathrm{L}$ & 417 & $6.25[4.98-8.83]$ & $5.92[4.77-7.82]$ & $6.44[5.06-8.95]$ & 0.10 \\
\hline Neutrophils, $10^{9} / \mathrm{L}$ & 376 & $4.74[3.55-7.21]$ & $4.52[3.55-6.50]$ & $4.87[3.55-7.32]$ & 0.50 \\
\hline Neutrophils, \% & 376 & $77.7[70.5-84.7]$ & $76.6[69.7-84.2]$ & $78.0[71.3-84.8]$ & 0.54 \\
\hline Lymphocytes, $10^{9} / \mathrm{L}$ & 263 & $0.89[0.58-1.23]$ & $0.89[0.62-1.13]$ & $0.90[0.58-1.24]$ & 0.68 \\
\hline Lymphocytes, \% & 263 & $14.0[7.9-19.5]$ & $14.6[8.7-19.9]$ & $13.9[7.6-19.3]$ & 0.56 \\
\hline Monocytes, \% & 263 & $6.1[3.9-8.1]$ & $5.2[3.2-7.9]$ & $6.2[4.2-8.3]$ & 0.048 \\
\hline Eosinophils, \% & 263 & $0.00[0.00-0.30]$ & $0.00[0.00-0.30]$ & $0.00[0.00-0.30]$ & 0.65 \\
\hline Basophils, \% & 263 & $0.20[0.10-0.30]$ & $0.20[0.10-0.30]$ & $0.20[0.10-0.30]$ & 0.75 \\
\hline Platelets, $10^{9} / \mathrm{L}$ & 407 & $179[140-226]$ & 209 [158-252] & 171 [136-212] & $<0.001$ \\
\hline$\geq 150$-no. (\%) & 407 & $290(71.3)$ & $94(83.9)$ & $196(66.4)$ & $<0.001$ \\
\hline INR, ratio & 353 & $1.07[1.02-1.16]$ & $1.05[1.01-1.12]$ & $1.08[1.02-1.16]$ & 0.037 \\
\hline aPTT, ratio & 338 & $1.15[1.04-1.29]$ & 1.09 [0.99-1.27] & $1.17[1.06-1.30]$ & 0.024 \\
\hline AST, U/L & 410 & $49.0[35.0-74.0]$ & $40.0[29.0-55.0]$ & $53.0[38.0-84.0]$ & $<0.001$ \\
\hline $\mathrm{ALT}, \mathrm{U} / \mathrm{L}$ & 412 & $37.0[25.0-59.0]$ & $30.0[20.0-45.0]$ & $40.0[27.0-62.0]$ & $<0.001$ \\
\hline Total bilirubin, mg/dL & 372 & $0.60[0.50-0.90]$ & $0.60[0.40-0.80]$ & $0.70[0.50-0.90]$ & 0.020 \\
\hline Creatinine, mg/dL & 414 & $0.92[0.77-1.23]$ & $0.74[0.61-0.91]$ & 0.98 [0.84-1.35] & $<0.001$ \\
\hline Urea, mg/dL & 354 & $45.0[34.0-66.0]$ & $38.0[28.0-55.0]$ & $48.0[37.0-71.0]$ & $<0.001$ \\
\hline $\mathrm{LDH}, \mathrm{U} / \mathrm{L}$ & 371 & 384 [300-516] & 368 [291-484] & 391 [308-529] & 0.13 \\
\hline D-dimer, ng/mL & 13 & 2337 [1587-6028] & 4182 [1864-11437] & 2067 [1463-3328] & 0.32 \\
\hline Fibrinogen, g/L & 94 & $0.61[0.49-0.71]$ & $0.53[0.48-0.66]$ & $0.63[0.49-0.72]$ & 0.19 \\
\hline CRP, mg/L & 417 & 113 [56-16.2] & 102 [48-145] & 122 [58-165] & 0.051 \\
\hline$\geq 127$-no. (\%) & 417 & $188(45.1)$ & $40(35.4)$ & $148(48.7)$ & 0.015 \\
\hline $\mathrm{PCT}, \mathrm{ng} / \mathrm{mL}$ & 84 & $0.49[0.12-1.90]$ & $0.12[0.05-1.00]$ & $0.56[0.21-2.35]$ & 0.019 \\
\hline $\mathrm{Na}, \mathrm{mEq} / \mathrm{L}$ & 414 & 138 [136-141] & 138 [136-140] & 138 [136-141] & 0.86 \\
\hline $\mathrm{K}, \mathrm{mEq} / \mathrm{L}$ & 412 & $3.9[3.6-4.3]$ & $3.8[3.5-4.2]$ & $4.0[3.7-4.3]$ & 0.028 \\
\hline $\mathrm{Cl}, \mathrm{mEq} / \mathrm{L}$ & 289 & 102 [99-104] & 101 [98-103] & 102 [99-104] & 0.46 \\
\hline $\begin{array}{l}\text { Abnormalities at chest } \\
\text { X-ray—no. (\%) }\end{array}$ & 413 & $355(86.0)$ & $98(87.5)$ & $257(85.4)$ & 0.58 \\
\hline Unilateral & 413 & 77 (18.6) & $17(15.2)$ & $60(19.9)$ & 0.27 \\
\hline Bilateral & 413 & $278(67.3)$ & 81 (72.3) & $197(65.4)$ & 0.19 \\
\hline
\end{tabular}

Data expressed as median and IQR [Interquartile Range] (continuous variables) and as column percentages (categorical variables). Percentages may not total 100 because of rounding, Hemocromocytometric values data shown as Hemoglobin, White blood cells and Platelets count. INR = International Normalization Ratio for prothrombin time; aPTT = Activated Partial Thromboplastin Time; AST = Aspartate Aminotransferase; ALT = Alanine Aminotransferase; LDH = Lactate Dehydrogenase; $\mathrm{D}$-dimer $=$ Fibrin Degradation product; $\mathrm{CRP}=\mathrm{C}$-reactive Protein; $\mathrm{PCT}=$ Procalcitonin; $\mathrm{Na}=$ Sodium; $\mathrm{K}=\mathrm{Potassium} ; \mathrm{Cl}=\mathrm{Chloride} . \mathrm{p}$ values obtained by $\mathrm{Chi}$-square test (or Fisher's exact test when appropriate) for categorical variables and Wilcoxon-Mann-Whitney test for continuous variables

a third of hospitalized male population. Second, both 28-day mortality and severe disease occur less frequently in women. Third, different mortality in sex categories cannot be ascribed to age per se. Fourth, once severe disease has occurred, the risk of dying from Covid-19 is not affected by gender.

The importance of the evaluation of sex- and genderspecific effects of Covid-19 has been recently emphasized, with the aim to develop approaches able to address the acute and long-term effect of the disease [19]. Availability and access to health care facilities, especially in low income countries, could be different for women and men [20]. As largely expected, in our cohort, we do not find any significant difference between gender categories in terms of pre hospital antibiotic treatment, home or professional exposure to confirmed Covid-19 cases and interval between symptoms onset and hospital presentation, suggesting an equitable access to healthcare. Our study population mainly consists of male individuals (72.4\% vs $27.6 \%$ ), with a male/female ratio of $2.6: 1$. Available Covid-19 literature shows variable sex prevalence depending on clinical setting. Epidemiological reports based on notification of infectious disease describe similar prevalence between sex categories [12, 21, 22]. On 


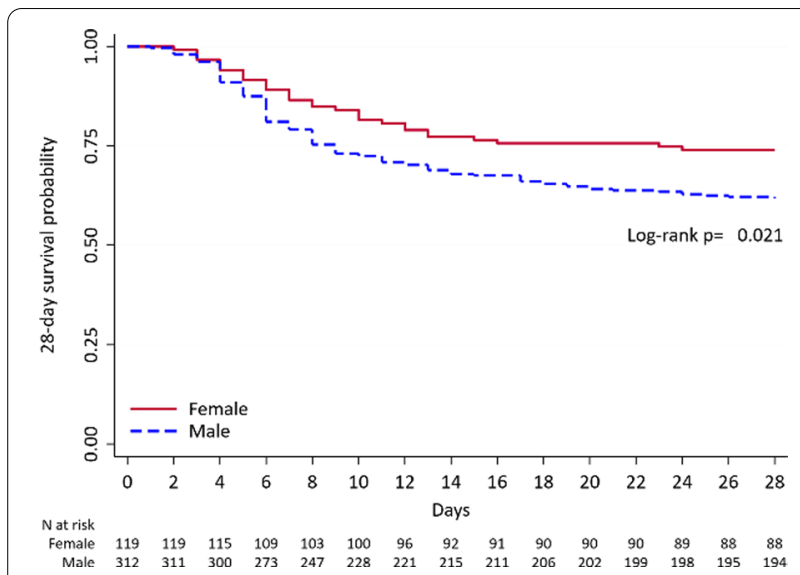

Fig. 1 Kaplan-Meier 28-day mortality since hospitalization by gender in the overall population the other hand, when considering hospitalized Covid19 population, a ratio of about $1.5: 1$ is found $[9,10,23]$. Moreover, male prevalence increases in ICU setting, ranging from 1.5 to 2.0:1 up to 4:1 in a recent Italian study [24-26]. Taken together, our study and other epidemiological data confirm a more severe disease in males. Furthermore, as a clue of this result, we found an exclusive use of IL- 6 inhibitors only in few males with particular compromised clinical condition and relentless deterioration in gas exchanges in spite of optimized conventional therapy (Table 3). Considering mortality, women are significantly more likely than men to survive the infection, in accordance with recent literature on Covid-19 [9]. Of note, in order to standardize and valorise the analysis, we described mortality at 28-day since hospitalization, which is a shared and reasonable interval of time in acute settings.

Table 5 Univariate and multivariable predictors of 28-day mortality since hospitalization

\begin{tabular}{|c|c|c|c|c|}
\hline & \multicolumn{2}{|c|}{ Univariate model (all candidate predictors) } & \multicolumn{2}{|c|}{ Multivariable model (selected predictors*) } \\
\hline & OR $(95 \% \mathrm{Cl})$ & $p$ & OR $(95 \% \mathrm{Cl})$ & $p$ \\
\hline Male sex & $1.75(1.10-2.80)$ & 0.019 & $1.05(0.47-2.37)$ & 0.898 \\
\hline \multicolumn{5}{|l|}{ Age $-y$} \\
\hline$\leq 59$ & 1.00 (Ref.) & - & 1.00 (Ref.) & - \\
\hline $60-69$ & $3.19(1.40-7.29)$ & 0.006 & $2.84(0.91-8.86)$ & 0.072 \\
\hline $70-77$ & $8.99(4.14-19.54)$ & $<0.001$ & $13.36(4.39-40.60)$ & $<0.001$ \\
\hline$\geq 78$ & $19.59(8.89-43.15)$ & $<0.001$ & $31.73(9.29-108.44)$ & $<0.001$ \\
\hline Former smoker & $2.17(1.31-3.58)$ & 0.003 & - & - \\
\hline Hypertension & $3.09(2.00-4.78)$ & $<0.001$ & - & - \\
\hline \multicolumn{5}{|l|}{ Diabetes } \\
\hline No diabetes & 1.00 (Ref.) & - & - & - \\
\hline Diabetes, not insulin dependent & $1.76(0.98-3.13)$ & 0.057 & - & - \\
\hline Diabetes, insulin dependent & $3.51(1.52-8.08)$ & 0.003 & - & - \\
\hline Chronic Kidney Failure & $2.56(1.22-5.36)$ & 0.012 & - & - \\
\hline COPD & $1.98(1.04-3.79)$ & 0.039 & - & - \\
\hline Active hematologic malignancy & $5.41(1.41-20.72)$ & 0.014 & - & - \\
\hline Previous Myocardial Infarction & $3.23(1.79-5.83)$ & $<0.001$ & - & - \\
\hline Vasculopathy & $3.18(1.78-5.68)$ & $<0.001$ & - & - \\
\hline ACE-inhibitors & $2.09(1.25-3.49)$ & 0.005 & - & - \\
\hline OAT/DOACS & $1.96(1.08-3.54)$ & 0.026 & - & - \\
\hline Antiplatelets & $3.10(1.98-4.87)$ & $<0.001$ & - & - \\
\hline Immunosuppression & $2.20(1.01-4.82)$ & 0.048 & - & - \\
\hline Flu vaccine & $2.53(1.51-4.25)$ & $<0.001$ & - & - \\
\hline Symptoms onset-ER, per 1-day increase & $0.92(0.87-0.97)$ & 0.001 & - & - \\
\hline Cough & $0.61(0.40-0.91)$ & 0.016 & - & - \\
\hline Dyspnea before admission & $1.66(1.09-2.52)$ & 0.018 & - & - \\
\hline Systemic symptoms & $0.61(0.39-0.96)$ & 0.032 & - & - \\
\hline CPAP/NIV in the first $24 \mathrm{~h}$ & $4.73(2.97-7.53)$ & $<0.001$ & $7.07(3.20-15.61)$ & $<0.001$ \\
\hline $\mathrm{PaO}_{2} / \mathrm{FiO}_{2}$ ratio $<200$ & $3.73(2.26-6.14)$ & $<0.001$ & $3.05(1.49-6.24)$ & 0.002 \\
\hline Blood urea, per 1-unit increase & $1.02(1.01-1.02)$ & $<0.001$ & $1.01(1.00-1.02)$ & 0.041 \\
\hline
\end{tabular}




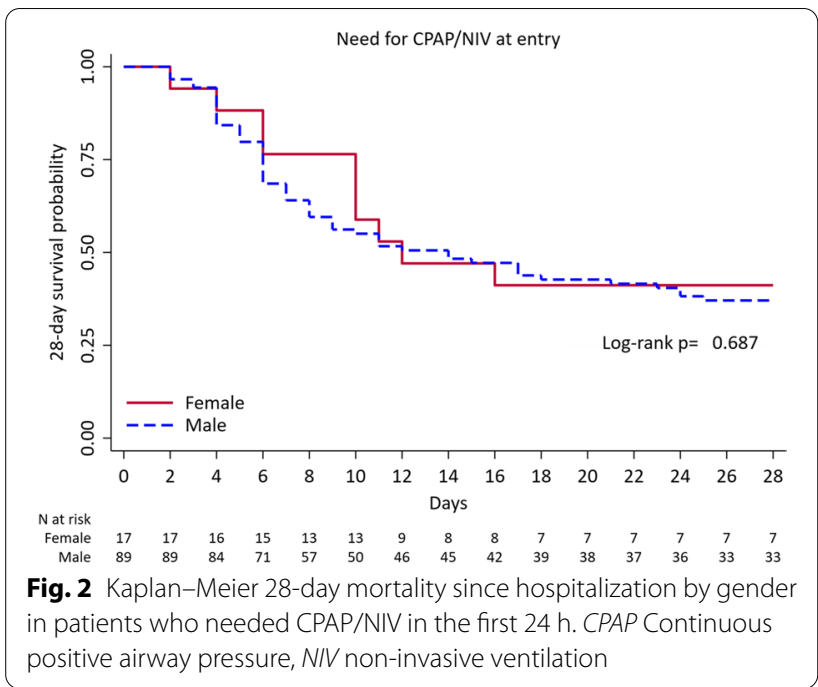

In the case of Covid-19, an enzymatic system involved in this different sex predisposition could be represented by angiotensin converting enzyme 2 (ACE2), which allows penetration of SARS-CoV-2 into cells and is downregulated by the virus [13]. ACE2 is counter regulatory to the activity of angiotensin II, leading to angiotensin-(1-7) formation, which exerts vasodilatory, anti-inflammatory, antifibrotic, and antigrowth effects. Animal model observations demonstrated a hormonal susceptibility of ACE2. In mice it has been shown that $17 ß$-estradiol increases the expression and activity of ACE2 while ovariectomy results in a decreased activity. Conversely hypertensive male mice have a higher myocardial ACE2 expression than females and its levels decreases after orchiectomy $[27,28]$. Moreover, sex hormones can affect the immune and inflammatory modulation during infection, with estrogens promoting both innate and adaptive immunity and testosterone having a suppressive effect on immune function [29]. Actually, in our cohort, biochemical profile at presentation (i.e. platelets counts, coagulation, liver and renal function, CRP and PCT) suggests a tendency to a lower inflammatory status and organs impairment in females (Table 4). Finally, preliminary data have advocated a crucial role of endothelium in Covid-19. A role of estrogen (i.e. 17ß-estradiol or E2) on vascular function and the endothelium have been suggested [30]. The mechanisms proposed include the generation of $\mathrm{NO}$ and prostacyclin, promotion of endothelial repair and regeneration, anti-inflammatory and antioxidant effects [31]. Our female population confirmed to be less fragile in this field, having few lifestyle risk factors (i.e. smoking history), and a lower rate of vasculopathy and myocardial infarction (Table 1). Thus, the lower severity of Covid-19 in women can be due to the influence of gender-related factors at least on: (1) the mechanism of cell entry of the virus; (2) the immune and inflammatory modulation during infection; (3) the endothelium and vascular function. Moreover, gastrointestinal symptoms at presentation, which were inconsistently correlated to outcome in previous reports, are more common in females (Table 2) [32-35]. This result could reflect the higher expression of ACE2 in colon transverse in females. As a matter of fact, a recent systematic survey showed that ACE2 presents remarkable differences in male-female expression levels possibly due to differences in escape from $\mathrm{X}$ inactivation [36].

Interestingly, in our multivariable analysis, sex does not result an independent predictor of death. Similar results were found during SARS in 2003, where a significantly higher mortality rate in males have been described, even if sex was not an independent predictor of mortality [37]. Specifically, when we add the severity of respiratory failure at presentation in the multivariable model (i.e. $\mathrm{PaO}_{2} /$ $\mathrm{FiO}_{2}$ ratio $<200 \mathrm{mmHg}$ at presentation and CPAP/NIV need in the first $24 \mathrm{~h}$ ), it prevails on sex influence. The multivariable model is confirmed by survival analysis which demonstrates that male and female patients who required CPAP or NIV in the first $24 \mathrm{~h}$ have similar outcome (Fig. 2).

Our study has several limitations. First, it must be acknowledged that this is a retrospective study based on electronic medical records collected during a medical emergency, thus the accuracy of data may be reasonably questioned. We cannot exclude that missing data could have affected the significance of some variables. However, the effect of gender on mortality risk did not change (OR male vs. female $=1.39,95 \%$ CI $0.76-2.45$, $p=0.288)$ if we imputed missing values using multiple imputation by chained equation (MICE) with 20 imputation sets. Of note, this is a large series of cases coming from the forefront of the outbreak, addressing gender differences and providing a substantial follow-up length on hard endpoints. Secondly, our cohort consists of a large proportion of male patients, and this could have brought to an imbalance between represented sex categories. Moreover, research on sex and gender exceeds stratification of patients by these variables, needing the evaluation of biological (e.g. hormonal state, immune function and comorbidities), and gender-related factors (e.g. lifestyle and socioeconomic status) [19]. Prospective studies are required to better characterize patients by the evaluation of more specific sex- and gender-related parameters.

\section{Conclusions}

Women admitted for Covid-19 have significantly few cardiovascular comorbidities and lifestyle risk factors, and more gastrointestinal symptoms prior to admission. 
Moreover, female patients present less severe disease and are more likely to survive the infection. However, once severe disease occurs, the risk of dying is similar regardless of gender.

\begin{abstract}
Abbreviations
ABG: Arterial blood gas analysis; ACE2: Angiotensin converting enzyme 2; ALT: Alanine aminotransferase; AST: Aspartate transaminase; CCl: Charlson Comorbidity Index; Cl: Confidence intervals; Covid-19: Coronavirus disease 2019; CPAP: Continuous positive airway pressure; CRP: C Reactive protein; E2: Estradiol; ER: Emergency room; ETI: Endotracheal intubation; $\mathrm{FiO}_{2}$ : Fractional inspired oxygen; $\mathrm{HCO}_{3}{ }^{-}$: Bicarbonate; HIV: Human immunodeficiency virus; ICU: Intensive Care Unit; IL-6: Interleukin-6; IQR: Interquartile range; MERS: Middle east respiratory syndrome; MICE: Multiple imputation by chained equation; NIV: Non-invasive ventilation; $\mathrm{NO}$ : Nitric oxide; OR: Odds ratio; $\mathrm{PaCO}_{2}$ : Partial pressure of carbon dioxide; $\mathrm{PaO}_{2}$ : Arterial oxygen partial pressure; $\mathrm{PCT}$ : Procalcitonin; RNA: Ribonucleic acid; RT-PCR: Real time polymerase chain reaction; SARS: Severe acute respiratory syndrome; SARS-CoV-2: Severe acute respiratory syndrome coronavirus 2; SD: Standard deviation; WHO: World Health Organization.
\end{abstract}

\section{Supplementary Information}

The online version contains supplementary material available at https://doi. org/10.1186/s12890-021-01455-0.

Additional file 1: Supplementary Definitions. Table S1. Intervals between symptoms and clinical relevant episodes in all patients and by gender. Table S2. 28-day outcomes according to gender and age.

Additional file 2. Covid-19 Study Group.

\section{Acknowledgements}

We would like to acknowledge all the members of HPG23 Covid-19 Study Group listed in Additional file 2.

\section{Authors' contributions}

$F R$, $L N$, and FDM conceived the idea and designed the research. FR, LN, FMR, RB, RT, LGi, MAn, MAm, CA, GI, CMS, SCo, EH, LM, LGa, PM, MM collected clinical records data. AC and CF performed and supervised all molecular and biological analyses. AGh analysed study data and developed statistical models and design of methodology. FDM and SF were the responsible for the research activity, management and coordination. FR, LN, FDM, and AGh created and wrote the initial draft. All the authors critically analysed data and revised the draft. FR, LN and FDM prepared the final version of manuscript after revision and final approval by all the authors.

\section{Funding}

None.

\section{Availability of data and materials}

The datasets generated and/or analysed during the current study are available in Papa Giovanni XXIII Hospital digital repository. The datasets generated and/ or analysed during the current study are not publicly available due individual privacy policy but are available from the corresponding author on reasonable request.

\section{Declarations}

\section{Ethics approval and consent to participate}

This retrospective, observational study was approved by the local Ethics Committee (Comitato Etico di Bergamo, Italy. N³7/2020). According to the approved protocol, verbal consent was obtained when feasible. We avoided written consent in the light of the urgent need to treat critical patients, and to avoid paper contamination. The accepting physician was responsible for verbal consent collection, it was documented by signing a declaration form.
Consent for publication

Not applicable.

\section{Competing interests}

The authors declare that they have no competing interests.

\section{Author details}

${ }^{1}$ Pulmonary Medicine Unit, Medicine Department, ASST Papa Giovanni XXIII, Piazza OMS, 1, 24127 Bergamo, Italy. ${ }^{2}$ Università degli Studi di Milano, Milan, Italy. ${ }^{3}$ FROM Research Foundation, Bergamo, Italy. ${ }^{4}$ Intensive Care Unit, ASST Papa Giovanni XXIII, Bergamo, Italy. ${ }^{5}$ Cardiovascular Department, ASST Papa Giovanni XXIII, Bergamo, Italy. ${ }^{6}$ Infectious Diseases Unit, ASST Papa Giovanni XXIII, Bergamo, Italy. ${ }^{7}$ Department of Oncology and Hematology, ASST Papa Giovanni XXIII, Bergamo, Italy. ${ }^{8}$ Emergency Department, ASST Papa Giovanni XXIII, Bergamo, Italy. ${ }^{9}$ Department of Organ Failure and Transplantation, ASST Papa Giovanni XXIII, Bergamo, Italy. ${ }^{10}$ Department of Health and Social Care Professions, ASST Papa Giovanni XXIII, Bergamo, Italy. ${ }^{11}$ Neurology Unit, ASST Papa Giovanni XXIII, Bergamo, Italy. ${ }^{12}$ Quality Management, ASST Papa Giovanni XXIII, Bergamo, Italy. ${ }^{13}$ Mario Negri Institute for Pharmacological Research IRCCS, Anna Maria Astori Centre, Science and Technology Park Kilometro Rosso, Bergamo, Italy. ${ }^{14}$ Nephrology Unit, ASST Papa Giovanni XXIII, Bergamo, Italy. ${ }^{15}$ Microbiology and Virology Unit, ASST Papa Giovanni XXIII, Bergamo, Italy. ${ }^{16}$ Pathology Unit, ASST Papa Giovanni XXIII, Bergamo, Italy. ${ }^{17}$ Department of Research, Innovation, Brand Reputation, ASST Papa Giovanni XXIII, Bergamo, Italy. ${ }^{18}$ Department of Diagnostic Radiology, ASST Papa Giovanni XXIII, Bergamo, Italy. ${ }^{19}$ Università degli Studi di Milano-Bicocca, Milan, Italy. ${ }^{20}$ Gastroenterology Hepatology and Transplantation Unit, ASST Papa Giovanni XXIII, Bergamo, Italy.

Received: 12 June 2020 Accepted: 3 March 2021

Published online: 20 March 2021

\section{References}

1. World Health Organization (WHO). Statement on the Covid-19 situation in Europe. http://www.euro.who.int/en/health-topics/health-emerg encies/coronavirus-covid-19/statements/statement-on-the-covid-19situation-in-europe. Last visited 20 May, 2020.

2. Italian Institute of Statistics (ISTAT). Impact of Covid-19 on overall mortality during the first trimester of 2020. https://www.istat.it/it/files//2020/05/ Rapporto_Istat_ISS.pdf. Last visited 20 May, 2020.

3. La Vignera S, Cannarella R, Condorelli RA, et al. Sex-specific SARS-CoV-2 mortality: among hormone-modulated ACE2 expression, risk of venous thromboembolism and hypovitaminosis D. Int J Mol Sci. 2020;21:2948. https://doi.org/10.3390/ijms21082948.

4. Wenham C, Smith J, Morgan R, et al. COVID-19: the gendered impacts of the outbreak. Lancet. 2020;395:846-8. https://doi.org/10.1016/S01406736(20)30526-2.

5. Anna Ruggieri MCG. Gender differences in COVID-19: some open questions. Ital J Gender-Specif Med. 2020;6:49-50.

6. Sharma G, Volgman AS, Michos ED. Sex differences in mortality from COVID-19 pandemic: are men vulnerable and women protected? JACC Case Rep. 2020. https://doi.org/10.1016/j.jaccas.2020.04.027.

7. Jin J-M, Bai P, He W, Wu F, Liu X-F, Han D-M, Liu S, Yang J-K. Gender differences in patients with COVID-19: focus on severity and mortality. Front Public Health. 2020;8:152. https://doi.org/10.3389/fpubh.2020.00152.

8. Karlberg J, Chong DS, Lai WY. Do men have a higher case fatality rate of severe acute respiratory syndrome than women do? Am J Epidemiol. 2004;159:229-31. https://doi.org/10.1093/aje/kwh056.

9. Mehra MR, Desai SS, Kuy S, et al. Cardiovascular disease, drug therapy, and mortality in Covid-19. N Engl J Med. 2020. https://doi.org/10.1056/NEJMo a2007621.

10. Guan WJ, Ni ZY, Hu Y, et al. Clinical characteristics of coronavirus disease 2019 in China. N Engl J Med. 2019. https://doi.org/10.1056/NEJMoa2002 032.

11. Xie J, Tong Z, Guan X, et al. Clinical characteristics of patients who died of coronavirus disease 2019 in China. JAMA Netw Open. 2020;3:e205619. https://doi.org/10.1001/jamanetworkopen.2020.5619.

12. Covid-19 sex disaggregated data tracker. Sex, gender and Covid-19. http://globalhealth5050.org/covid19. Last visited 20 May, 2020. 
13. Zhou $P$, Yang $X L$, Wang XG, et al. A pneumonia outbreak associated with a new coronavirus of probable bat origin. Nature. 2020;579:270-3. https:// doi.org/10.1038/s41586-020-2012-7.

14. Channappanavar R, Fett C, Mack M, et al. Sex-based differences in susceptibility to severe acute respiratory syndrome coronavirus infection. J Immunol. 2017;198:4046-53. https://doi.org/10.4049/jimmunol.1601896.

15. Klein SL, Flanagan KL. Sex differences in immune responses. Nat Rev Immunol. 2016;16:626-38. https://doi.org/10.1038/nri.2016.90.

16. Gausman J, Langer A. Sex and gender disparities in the COVID-19 pandemic. J Women's Health. 2020;29:465-6. https://doi.org/10.1089/jwh. 2020.8472

17. World Health Organization. Coronavirus disease (COVID-19) outbreak. Interim guidance https://www.who.int/publications-detail/laboratorytesting-for-2019-novel-coronavirus-in-suspected-human-cases-20200 117. Last visited 20 May, 2020.

18. Corman VM, Landt O, Kaiser M, et al. Detection of 2019 novel coronavirus (2019-nCoV) by real-time RT-PCR. Euro Surveill. 2019;2020:25. https://doi. org/10.2807/1560-7917.ES.2020.25.3.2000045.

19. Spagnolo PA, Manson JE, Joffe H. Sex and gender differences in health: what the COVID-19 pandemic can teach us. Ann Intern Med. 2020. https://doi.org/10.7326/M20-1941.

20. Chiang C, Labeeb SA, Higuchi M, et al. Barriers to the use of basic health services among women in rural southern Egypt (Upper Egypt). Nagoya J Med Sci. 2013;75:225-31.

21. Novel Coronavirus Pneumonia Emergency Response Epidemiology Team. Vital surveillances: the epidemiological characteristics of an outbreak of 2019 novel coronavirus diseases (COVID-19) —China, 2020. China CDC Weekly. Accessed February 20, 2020. http://weekly.chinacdc.cn/en/artic le/id/e53946e2-c6c4-41e9-9a9b-fea8db1a8f51. Last visited 20 May, 2020.

22. Italian Health Institute (ISS). Covid-19 13 May 2020 report. https://www. epicentro.iss.it/coronavirus/bollettino/Infografica_13maggio\%20ITA.pdf. Last visited 20 May, 2020.

23. Richardson S, Hirsch JS, Narasimhan M, et al. Presenting characteristics, comorbidities, and outcomes among 5700 patients hospitalized with COVID-19 in the New York City area. JAMA. 2020. https://doi.org/10.1001/ jama.2020.6775.

24. Bhatraju PK, Ghassemieh BJ, Nichols M, et al. Covid-19 in critically ill patients in the Seattle region_case series. N Engl J Med. 2020. https:// doi.org/10.1056/NEJMoa2004500.

25. Yang $X, Y u Y, X u$ J, et al. Clinical course and outcomes of critically ill patients with SARS-CoV-2 pneumonia in Wuhan, China: a single-centered, retrospective, observational study. Lancet Respir Med. 2020;8:47581. https://doi.org/10.1016/\$2213-2600(20)30079-5.

26. Grasselli G, Zangrillo A, Zanella A, et al. Baseline Characteristics and Outcomes of 1591 Patients Infected With SARS-CoV-2 Admitted to ICUs of the Lombardy Region, Italy. JAMA. 2020. https://doi.org/10.1001/jama. 2020.5394.

27. Dalpiaz PL, Lamas AZ, Caliman IF, et al. Sex hormones promote opposite effects on ACE and ACE2 activity, hypertrophy and cardiac contractility in spontaneously hypertensive rats. PLoS ONE. 2015;10:e0127515. https:// doi.org/10.1371/journal.pone.0127515.

28. Gupte M, Thatcher SE, Boustany-Kari CM, et al. Angiotensin converting enzyme 2 contributes to sex differences in the development of obesity hypertension in C57BL/6 mice. Arterioscler Thromb Vasc Biol. 2012;32:1392-9. https://doi.org/10.1161/ATVBAHA.112.248559.

29. Bartz D, Chitnis T, Kaiser UB, et al. Clinical advances in sex- and genderinformed medicine to improve the health of all: a review. JAMA Intern Med. 2020. https://doi.org/10.1001/jamainternmed.2019.7194.

30. Knowlton AA, Lee AR. Estrogen and the cardiovascular system. Pharmacol Ther. 2012;135:54-70. https://doi.org/10.1016/j.pharmthera.2012.03.007.

31. Green DJ, Hopkins ND, Jones $\mathrm{H}$, et al. Sex differences in vascular endothelial function and health in humans: impacts of exercise. Exp Physiol. 2016;101:230-42. https://doi.org/10.1113/EP085367.

32. Jin X, Lian JS, Hu JH, et al. Epidemiological, clinical and virological characteristics of 74 cases of coronavirus-infected disease 2019 (COVID-19) with gastrointestinal symptoms. Gut. 2019. https://doi.org/10.1136/ gutjnl-2020-320926.

33. Wang D, Hu B, Hu C, et al. Clinical characteristics of 138 hospitalized patients with 2019 novel coronavirus-infected pneumonia in Wuhan, China. JAMA. 2019. https://doi.org/10.1001/jama.2020.1585.

34. Zhang JJ, Dong X, Cao YY, et al. Clinical characteristics of 140 patients infected with SARS-CoV-2 in Wuhan, China. Allergy. 2020. https://doi.org/ 10.1111/all.14238.

35. Zhou F, Yu T, Du R, et al. Clinical course and risk factors for mortality of adult inpatients with COVID-19 in Wuhan, China: a retrospective cohort study. Lancet. 2020;395:1054-62. https://doi.org/10.1016/S0140-6736(20) 30566-3.

36. Tukiainen T, Villani AC, Yen A, et al. Landscape of X chromosome inactivation across human tissues. Nature. 2017;550:244-8. https://doi.org/10. 1038 /nature24265.

37. Lee $\mathrm{N}$, Hui D, Wu A, et al. A major outbreak of severe acute respiratory syndrome in Hong Kong. N Engl J Med. 2003;348:1986-94. https://doi. org/10.1056/NEJMoă85.

\section{Publisher's Note}

Springer Nature remains neutral with regard to jurisdictional claims in published maps and institutional affiliations.
Ready to submit your research? Choose BMC and benefit from:

- fast, convenient online submission

- thorough peer review by experienced researchers in your field

- rapid publication on acceptance

- support for research data, including large and complex data types

- gold Open Access which fosters wider collaboration and increased citations

- maximum visibility for your research: over 100M website views per year

At BMC, research is always in progress.

Learn more biomedcentral.com/submissions 